\title{
A programming triangular DNA origami for doxorubicin loading and delivering to target ovarian cancer cells
}

\author{
Tao Song ${ }^{1,2}$, Xun Wang ${ }^{1}$, Xin $\mathrm{Li}^{4}$ and Pan Zheng ${ }^{3}$ \\ ${ }^{1}$ College of Computer and Communication Engineering, China University of Petroleum, Qingdao 266580, China \\ ${ }^{2}$ Departamento de Inteligencia Artificial, Universidad Politécnica de Madrid, Campus de Montegancedo, Boadilla del Monte \\ 28660, Spain \\ ${ }^{3}$ Faculty of Engineering, Computing and Science, Swinburne University of Technology Sarawak Campus, Kuching 93350, \\ Malaysia \\ ${ }^{4}$ Department of Gynecology 2, Renmin Hospital of Wuhan University, Wuhan 430060, China \\ Correspondence to: Xun Wang, email: wangsyun@upc.edu.cn \\ Keywords: DNA nanotechnology; DNA origami; drug cargo; ovarian cancer cells \\ Received: September 04, $2017 \quad$ Accepted: December 18, $2017 \quad$ Published: December 28, 2017 \\ Copyright: Song et al. This is an open-access article distributed under the terms of the Creative Commons Attribution License 3.0 \\ (CC BY 3.0), which permits unrestricted use, distribution, and reproduction in any medium, provided the original author and source \\ are credited.
}

\section{ABSTRACT}

DNA origamis provide plenty of platforms for constructing drug-delivery systems in nanoscale. In this work, we developed triangular DNA origami in 120nm around by M13mp18 DNA strand scaffold propagated in E. coli ER2738, which shows desired thermodynamic stability and well biocompatibility. It is tested the proposed DNA origami performance on loading doxorubicin, which is a chemotherapy medication used to treat cancer. In cell intervention experiments, the obtained complex with doxorubicin achieve acceptable performances on penetrating ovarian cancer cells and inhibiting production of A2780 ovarian cancer cells. This DNA nanotechnology provides an efficient nanoscale drug delivering systems or drug cargo system for inhibiting the reproduction of ovarian cancer cells in vitro.

\section{INTRODUCTION}

DNA nanotechnology is the design and manufacture of artificial nucleic acid structures for technological uses [1-5], such as molecular machine [6-8] and DNA computers [9-11]. In this field, DNA is used as nonbiological engineering materials for programming tools in nanotechnology. In DNA nanotechnology, DNA origami focuses on creating non-arbitrary two- and threedimensional nanostructures at the nanoscale [12].

In 2006, the scaffolder DNA origami technique is proposed and plenty of potential applications are suggested in [13]. One of the most important applications of DNA origami is for developing drug delivery or drug cargo systems. However, until 2010, most applications of DNA origamis are remain in a concept or testing phase [14]. Researchers focus on the design of DNA origami with different shape or functions, such as DNA origami tubes [15-16], square lattice [17-20], rectangular [21-22], triangular [17, 23-28] and so on.

Finding possible applications of DNA origamis, particularly in nanoscale, is becoming a promising branch in DNA nanotechnology. One of the most important one is taking or carrying drugs in molecular level to deliver them to targeting position in cell or tissue. This is due to the fact that DNA can be used as elemental materials to assemble functional structures, where molecular drugs can be embedded and deliver directly to targeting cells with help of DNA nanostructure to penetrating cells.

The general idea is to use DNA molecular as basic materials, such as bricks or tiles to form drug carriers. Since the cell has less rejection reaction to DNA, so the developed DNA nanostructure can help drugs entering cells. The concept is quite clear, but not easy to design and check the performance of DNA origamis. The first step should test the improvement of DNA origami to drugs in penetrating cells or cancer cells. 
In the recent five years, with the properties of penetrating cells well, drug loading capability, thermodynamic stability and well drug releasing efficiency, DNA origamis provide natural and feasible platforms for constructing drug-delivery systems in nanoscale. In 2017, it is formulated in [29] that the thermodynamic stability, cytotoxic effects, pharmacokinetic, drug loading and releasing capacities of DNA origami drug complex are formulated as four elemental challenges of DNA origami applications in cancer therapy. The size of DNA origami play vital roles in drug delivery: DNA origami of bigger sizes can take or load more drugs but decreasing their ability of penetrating cells. As well, the cost of assembling DNA origami of bigger sizes will increase heavily when a large number of carriers are needed to deliver drugs.

In [30], it is found that $\mathrm{A} 7 \mathrm{RC}$ peptides could enhance the uptake of vesicles by MDA-MB-231 cells, leading to stronger cytotoxicity in vitro and higher accumulation of vesicles in MDA-MB-231 xenografts in vivo, which make A7RC-LIPs be a promising drug carriers for anticancer. Adriamycin is frequently used in chemotherapy but it has cytotoxin. DNA nano structures designed as a carrier of Adriamycin can drastically reduce the toxic side effects and increase the circulation time [31]. Moreover, inserting Adriamycin into DNA template, using DNA as carrier is a new concept in DNA aptamer area. It is reported in [32] that Tamoxifen embedded in lipid bilayer improves the oncotarget of liposomal daunorubicin in vivo. An updated review paper on DNA origami based nanoscale drug deliver system can be found in [33].

In this work, we develop triangular DNA origami in $120 \mathrm{~nm}$ around by M13mp18 DNA strand scaffold propagated in E. coli ER2738. The proposed DNA origami triangular show desired thermodynamic stability by AFM scanning images, and also have good biocompatibility. The proposed triangular DNA origami is used to load doxorubicin, and achieving the complex of DNA triangular and doxorubicin. In cell intervention experiments, our complex has acceptable performances on penetrating ovarian cancer cells and inhibiting production of A2780 ovarian cancer cells $27 \%$ more than general doxorubicin. The proposed triangular DNA origami in $120 \mathrm{~nm}$ provides an efficient nanoscale drug delivering systems for inhibiting the reproduction of ovarian cancer cells in vitro with DNA nanotechnology.

\section{The design and thermodynamic stability of DNA triangular origami}

It starts by design DNA triangular origami in $120 \mathrm{~nm}$ by software caDNAno reported in [17] for designing twoand three- dimensional DNA origamis. Since caDNAno cannot calculate the thermodynamic properties, it needs spatial structure analysis and thermodynamic property calculating tools to evaluate the thermodynamic stability of designed DNA rectangular origami, and then select an optimal candidate. Here, Nanoengineer in [27] is applied for the three-dimensional spatial structure analysis of DNA origami, and the thermodynamic property can be calculated by submitting analyzing tasks to CanDo server [28]. Starting from a randomly designed DNA triangular origami, an optimal candidate is selected after thousands of negative and positive corrections by spatial structure and thermodynamic property analysis.

An overview of our designed DNA triangular origami in $120 \mathrm{~nm}$ drawing by caDNAno is shown in Figure 1. The shape of the DNA origami is determined by scaffold strand, which is in blue and composed of a certain number of small rectangular blocks. Each block is a double-strand DNA molecule, which consists of 8 basepairs. Scaffold strands are placed on rows above the ones with label even numbers, and staple strands (in different colors) are put on rows under the ones with label even numbers.

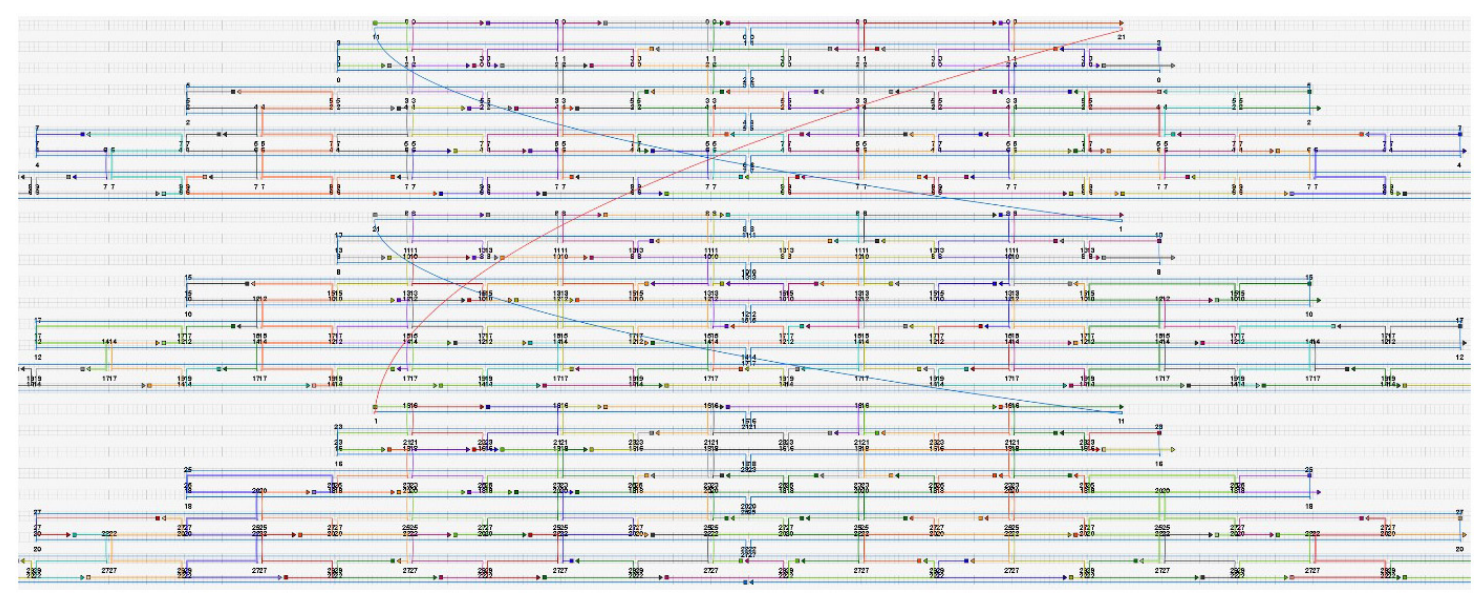

Figure 1: An overview design of DNA triangular origamis in 100nm by caDNAno. 
The three-dimensional structures and thermodynamic properties of DNA triangular origami in $120 \mathrm{~nm}$ by CanDo is shown in Figure 2, in which from blue to dark red, the thermodynamic stability of DNA origami is decrement. It is found in Figure 2 that the DNA origami triangular has good thermodynamic stability. Particularly, few parts are in red and these red parts have no essential effect on the shape of the DNA origami.

In Figure 3, AFM scanning images to DNA triangular origamis are shown. The image resolution is $500 \mathrm{~nm}$ with analyzing radius $100 \mathrm{~nm}$. These images show that the triangular DNA origamis have well thermodynamic stability for assembling.
The practical size of DNA triangular origami is of practical size with radius $123 \mathrm{~nm}$. The practical sizes are mostly consistent as they are theoretically designed, and the results are obtained by Brucker Section Analysis, shown in Figure 4.

\section{Doxorubicin loading rate analysis}

The doxorubicin loading rates is analyzed by absorption spectroscopy measurement. Specifically, the value of absorption spectroscopy of doxorubicin solution on the point $480 \mathrm{~nm}$ is set to be the initial concentration of doxorubicin, which is denoted by $E C_{\text {origin }}$. After
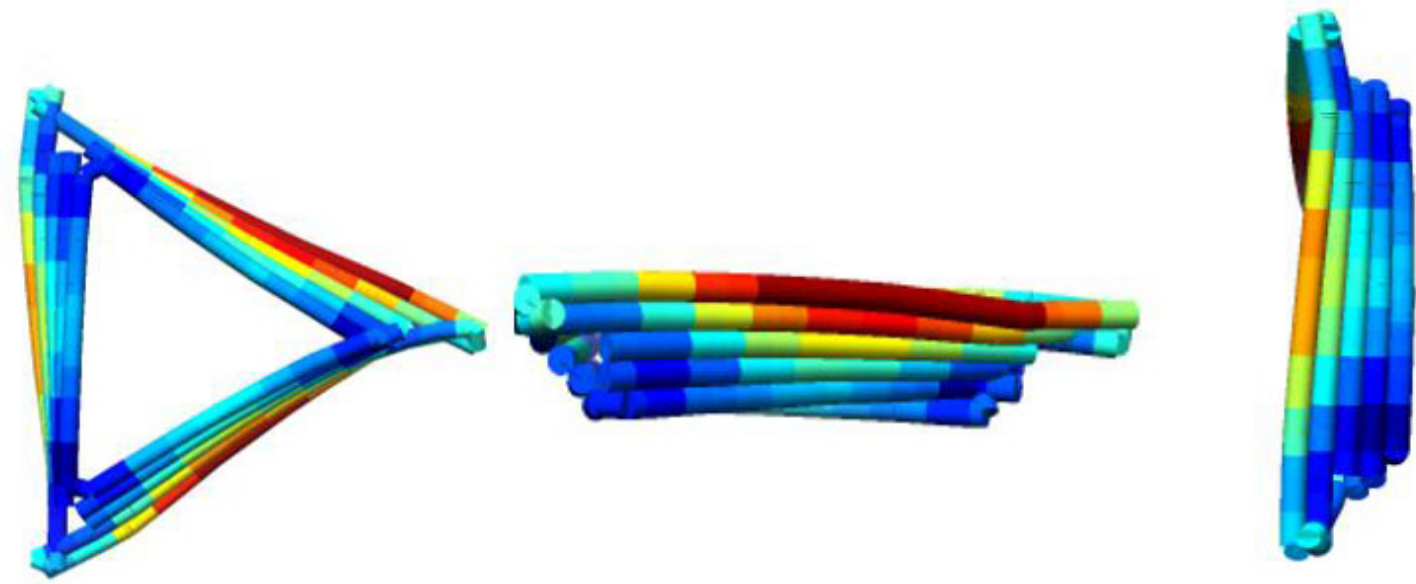

Figure 2: The three-dimensional structures and thermodynamic properties of DNA triangular origami in 120nm by CanDo.
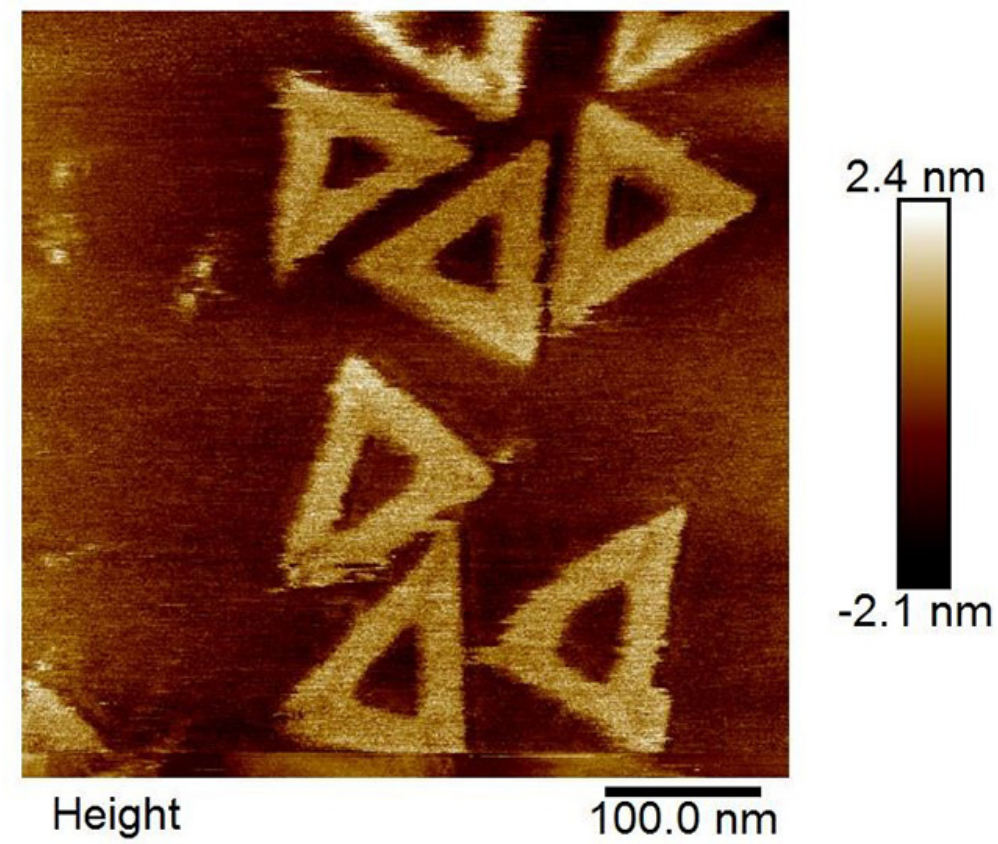

Figure 3: AFM scanning images of DNA rectangular and triangular origamis. 
Table 1: Absorption spectroscopy on 480nm with concentration of doxorubicin solution ranging from 0 to 2 with step-length $0.5 \mathrm{mM}$

\begin{tabular}{lccccccc}
\hline Dox Concentration $(\mathbf{m M})$ & $\mathbf{0}$ & $\mathbf{0 . 5}$ & $\mathbf{1}$ & $\mathbf{1 . 5}$ & $\mathbf{2}$ & Average & STDE \\
\hline 480nm absorption value & 0 & 0.2771 & 0.5583 & 0.9049 & 1.1075 & & \\
Extinction Coefficient & N/A & 0.5543 & 0.5583 & 0.6033 & 0.5538 & $\mathbf{0 . 5 6 7 3 7 5}$ & $\mathbf{0 . 0 0 0 8 8 6}$ \\
\hline
\end{tabular}

incubating with doxorubicin after certain hours, the $20 \mathrm{nM}$ absorption spectroscopy on the point $480 \mathrm{~nm}$ of doxorubicin solution left after purifying DNA origami doxorubicin complex by YM-100 centrifugal ultrafiltration is taken as concentration of remaining doxorubicin. It is written as $E C_{\text {extra }}$. The loading rate can be calculated by Equation (1).

$$
E C_{\text {load }}=\left[\left(E C_{\text {origin }}-E C_{\text {extra }}\right) / E C_{\text {origin }}\right] \times 100 \%
$$

The experiments on testing doxorubicin loading rates start with testing values of $E C_{\text {origin }}$ with concentration of doxorubicin solution ranging from $0 \mathrm{mM}$ to $2 \mathrm{mM}$ with step-length $0.5 \mathrm{mM}$. It is shown in Table 1 that the average value of Extinction Coefficient is 0.56737 with standard deviation (STDE) 0.000886 .

The doxorubicin solution concentration linear calibration curve on $480 \mathrm{~nm}$ is given Figure 5, where the correlation coefficient of R2 $=0.9998$.
We test loading rate of our designed DNA triangular origamis by incubating with doxorubicin in $12 \mathrm{~h}, 24 \mathrm{~h}, 48 \mathrm{~h}$ and $72 \mathrm{~h}$, respectively. All the samples are tested in $2 \mathrm{nM}$ doxorubicin solution. Doxorubicin loading rates of the DNA origamis are shown in Figure 6, where DNA double strands is in blue, and DNA triangular origami in size of $100 \mathrm{~nm}$ is in grey.

It is shown in Figure 6 that doxorubicin loading rate efficiency of DNA origamis increases gradually with time. Incubating with doxorubicin in 48 hours, our designed triangular DNA origami holds loading rate above $60 \%$, better than ordinary double stranded DNA loading rate (less than 30\%).

\section{Targeting A2780 ovarian cancer cells in vitro}

In this section, we analyze the biocompatibility of our designed triangular DNA origamis. As well, the performance of penetrating and inhibiting the production of A2780 ovarian cancer cell are tested.

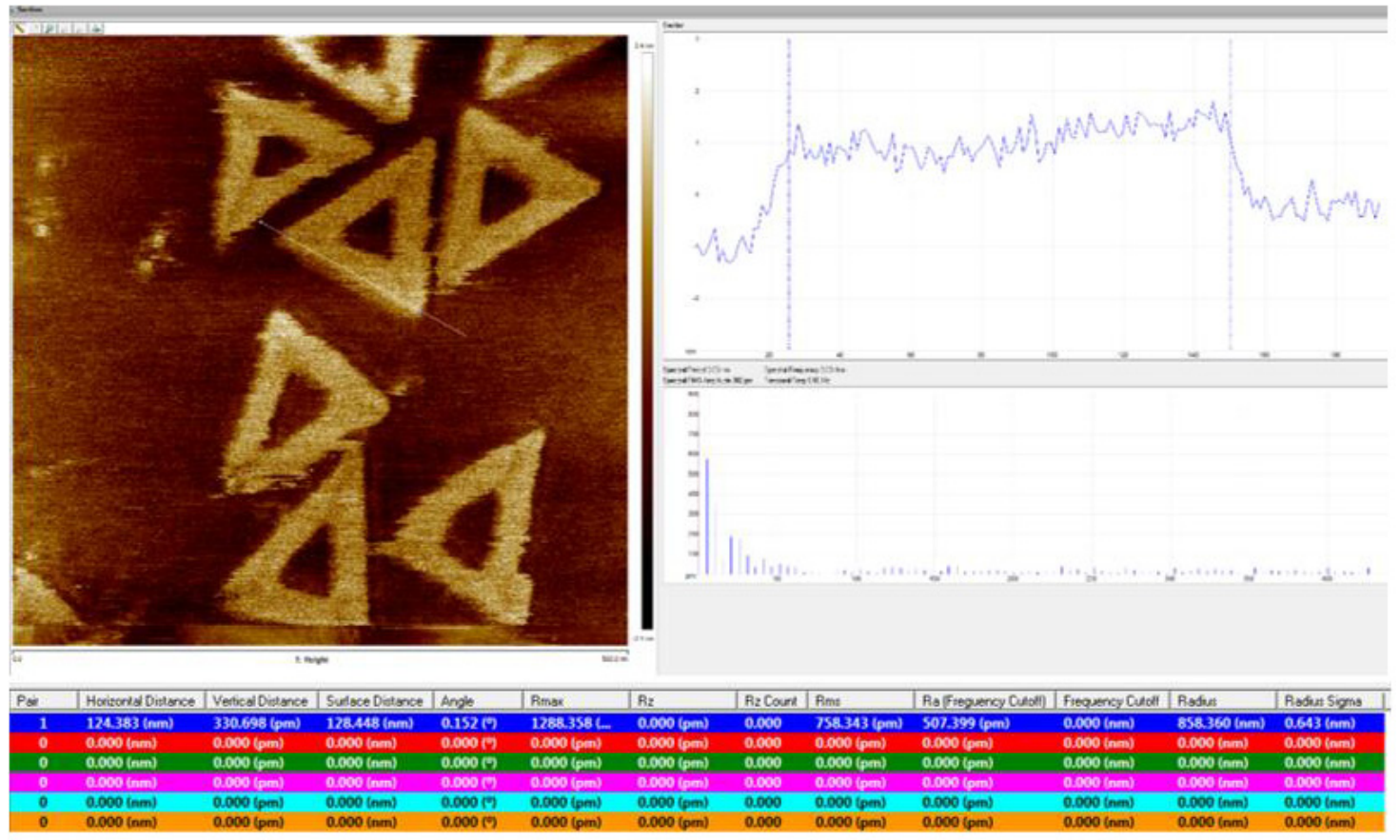

Figure 4: The practical size of DNA triangular origami with radius $123 \mathrm{~nm}$ by Brucker Section Analysis. 


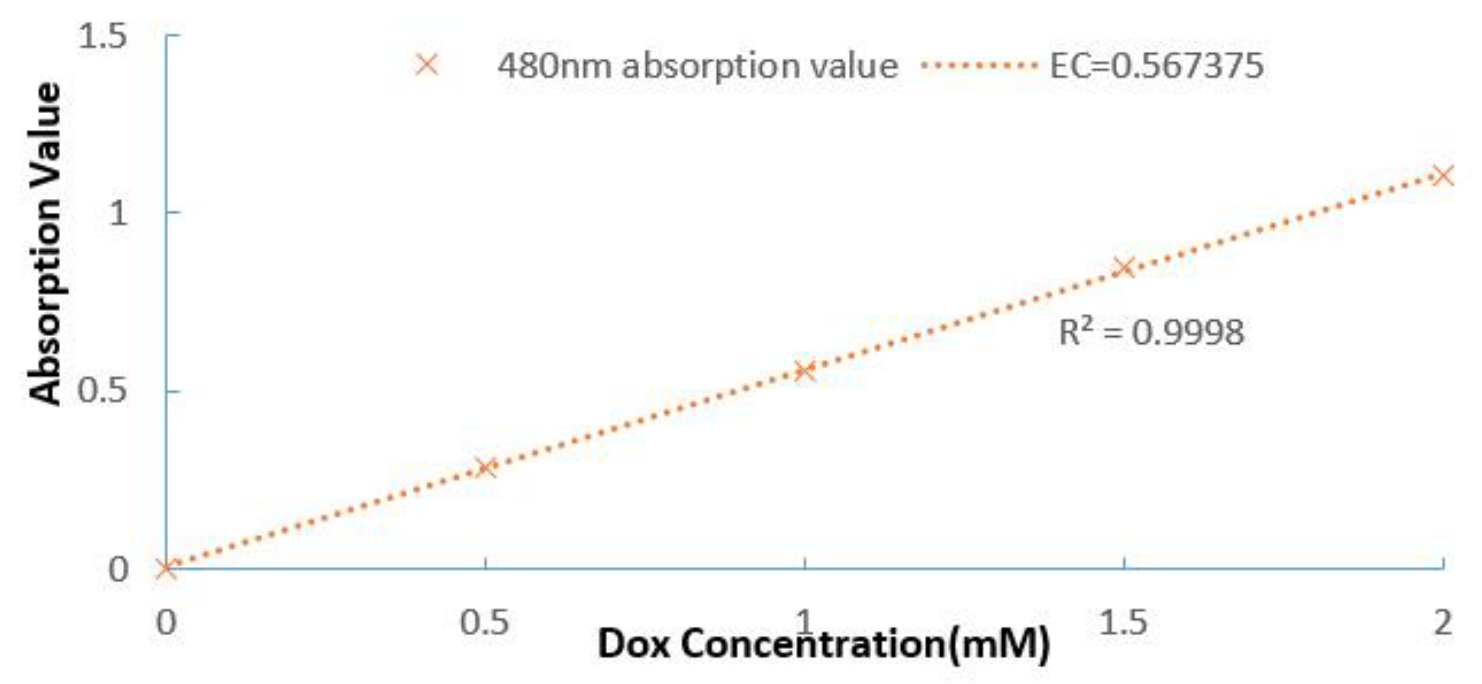

Figure 5: The doxorubicin solution concentration linear calibration curve on $480 \mathrm{~nm}$.

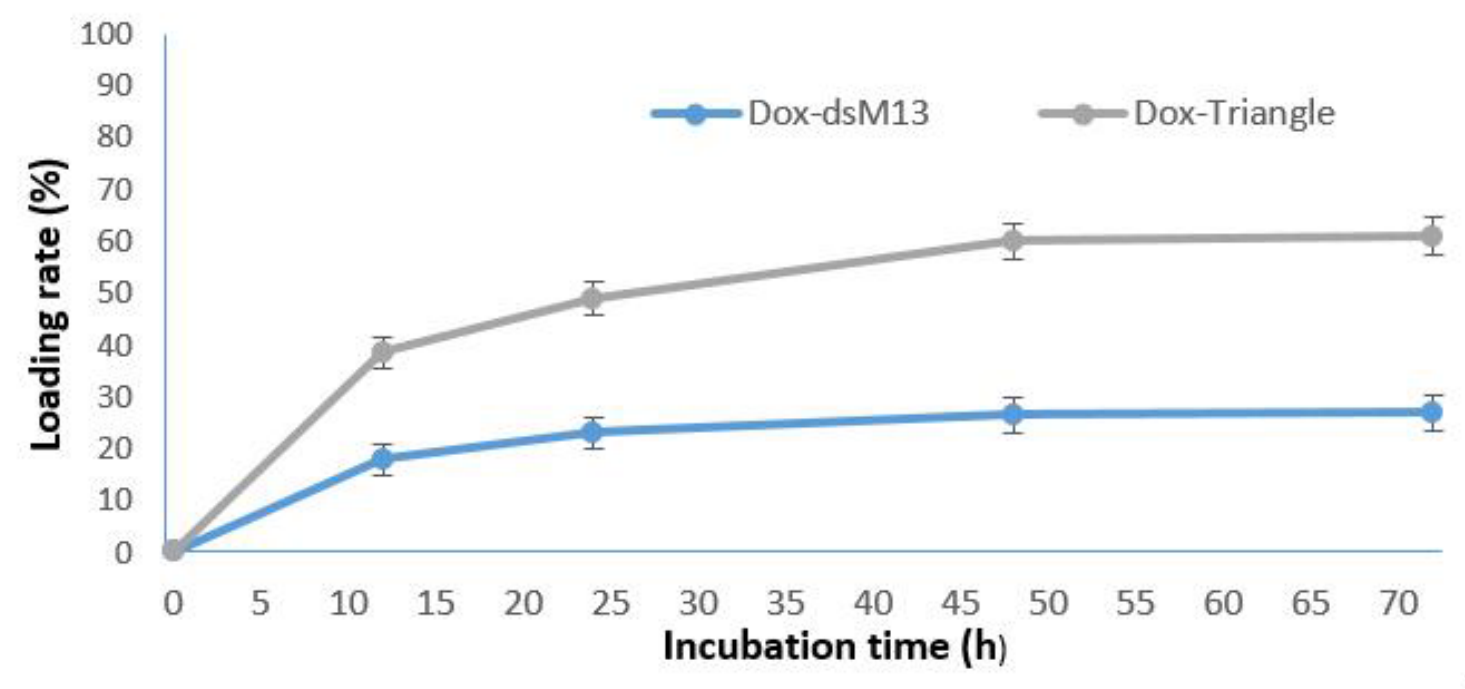

Figure 6: Loading rates of the DNA double strands and our designed DNA triangular origami.

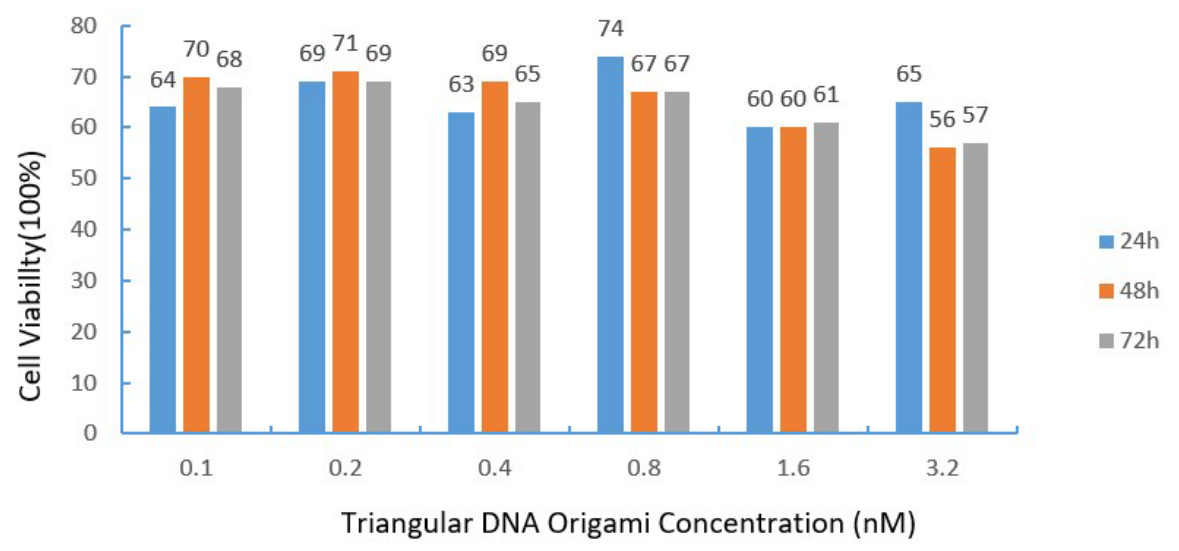

Figure 7: Cell viabilities by incubating triangular DNA origami into A2780 ovarian cancer cells solution in $24 \mathrm{~h}, 48 \mathrm{~h}$ and $72 \mathrm{~h}$ with concentrations ranging $0.1 \mathrm{nM}, 0.2 \mathrm{nM}, 0.4 \mathrm{nM}, 0.8 \mathrm{nM}, 1.6 \mathrm{nM}$ and $3.2 \mathrm{nM}$. 


\section{Analysis of biocompatibility}

The biocompatibility is tested by CCK-8 (Cell Counting Kit-8), which allows sensitive colorimetric assays for the determination of cell viability in cell proliferation and cytotoxicity assays. Here we show the biocompatibility tested by incubating the tested triangular DNA origamis in A2780 ovarian cancer cells solution in $24 \mathrm{~h}, 48 \mathrm{~h}$ and $72 \mathrm{~h}$, where the concentrations ranges are $0.1 \mathrm{nM}, 0.2 \mathrm{nM}, 0.4 \mathrm{nM}, 0.8 \mathrm{nM}, 1.6 \mathrm{nM}$ and $3.2 \mathrm{nM}$. It is shown in Figure 7, our designed triangular DNA origami has well biocompatibility with A2780 ovarian cancer cells, achieving cell viability above $65 \%$ by incubating into A2780 ovarian cancer cells solution in $24 \mathrm{~h}, 48 \mathrm{~h}$ and $72 \mathrm{~h}$.

Analysis of penetrating ability

Doxorubicin is a chemotherapy medication used to treat cancer, which is known to be fluorescent.
Doxorubicin fluorescence is quenched by binding to DNA. It is visible in fluorescence microscope by orangey-red in [29]. DAPI is a fluorescent stain that binds strongly to A-T rich regions in DNA, which is used extensively in fluorescence microscopy. It is shown by fluorescence microscope images in Figure 8 the uptake of $0.5 \mu \mathrm{M}$ triangular DNA origami loading doxorubicin and $0.5 \mu \mathrm{M}$ doxorubicin by A2780 ovarian cancer cells. This indicates our designed triangular DNA origami uptake doxorubicin performs better than doxorubicin penetrating into A2780 ovarian cancer cells, i.e., combined with DNA triangular origamis can help doxorubicin penetrating into cells.

It can be obtained from Figure 8 that with help of our designed triangular DNA origami, that is, as a doxorubicin delivering tool, doxorubicin can penetrate into cells with better performance.
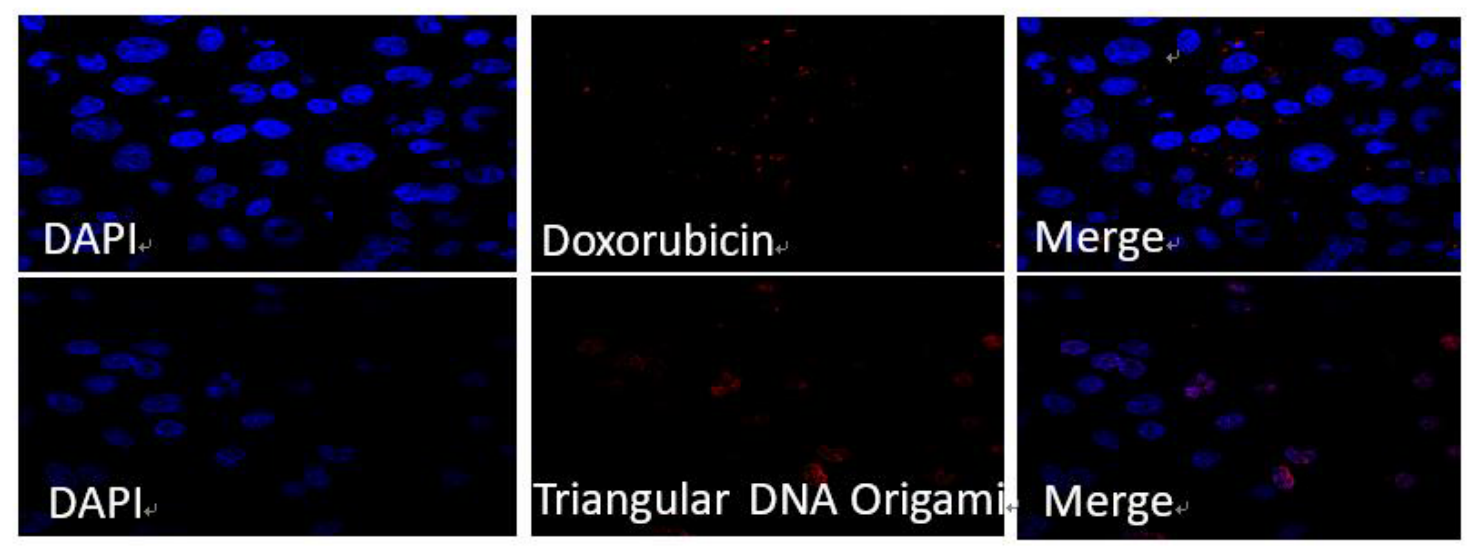

Figure 8: The fluorescence microscope images that the uptake of $0.5 \mu \mathrm{M}$ triangular DNA origami loading doxorubicin and $0.5 \mu \mathrm{M}$ doxorubicin by A2780 ovarian cancer cells in $36 \mathrm{~h}$.

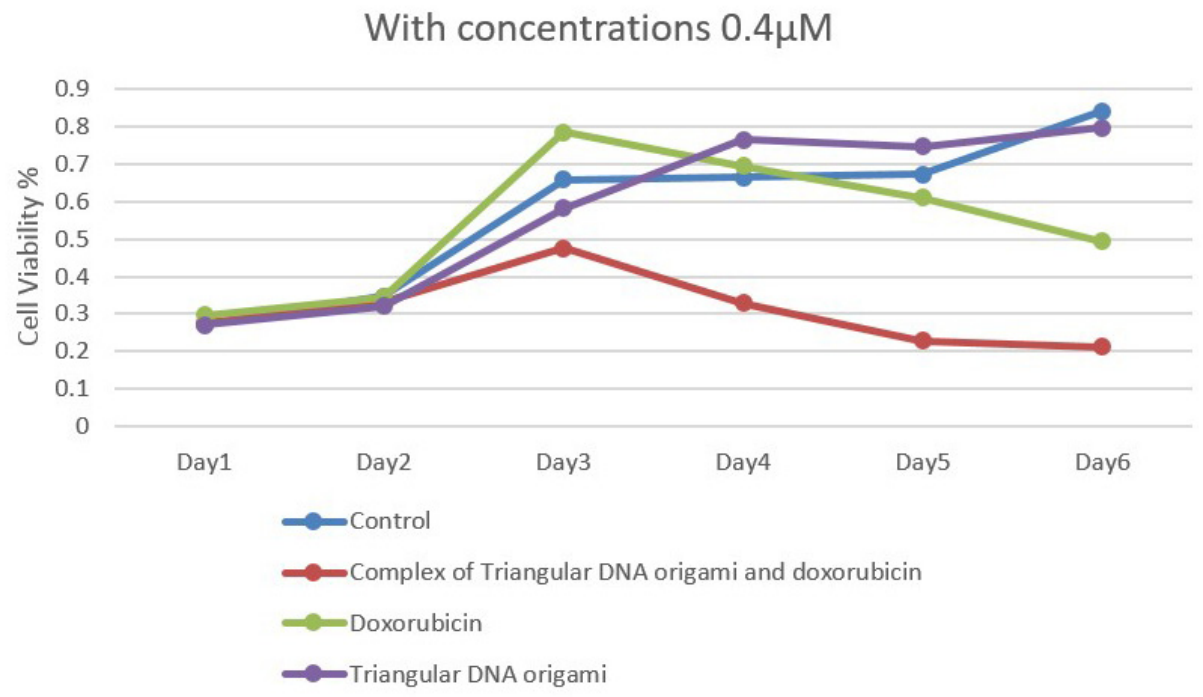

Figure 9: The cell proliferation by CCK-8 and inhibition of clone formation with concentration $0.4 \mu \mathrm{M}$. 


\section{Inhibiting A2780 ovarian cancer cells in vitro}

We test the inhibiting effect on the production of A2780 ovarian cancer cells by tracking the spectral absorptance of cells, which represents the numbers of cells, ranging from 1 day to 7 days in concentrations $0.4 \mu \mathrm{M}$ and $0.8 \mu \mathrm{M}$. The cell proliferation results by CCK8 and inhibition of clone formation are shown in Figures 9 and 10 .

It is obtained that the complex of our designed triangular DNA Origami and doxorubicin is much more efficient than doxorubicin in inhabiting A2780 ovarian cancer. With concentration $0.8 \mu \mathrm{M}, 30 \%$ more A2780 ovarian cancer cells can be inhibited in production in 7 days comparing with using only doxorubicin. If the concentration is set to be $0.4 \mu \mathrm{M}$, the inhibiting production of A2780 ovarian cancer cells is about $10 \%$ more than using only doxorubicin in 7 days.

\section{CONCLUSION AND DISCUSSION}

In this work, a programming triangular DNA origami in size of around $120 \mathrm{~nm}$ is developed with M13mp18 DNA strand scaffold propagated in E. coli ER2738. The proposed triangular DNA origamis are of size $123 \mathrm{~nm}$ by Brucker Section Analysis, which is accordance with the experimentally designed size. Our designed triangular DNA origami is with desired thermodynamic stability by AFM scanning images, and also perform well of having biocompatibility with
$65 \%$ cell viability. As a novel nanoscale drug cargo, the proposed triangular DNA origami can load doxorubicin at rate above $60 \%$, which is much higher than simple double DNA strands. It is obtained that the complex of our designed triangular DNA origami and doxorubicin is can target A2780 ovarian cancer cell, that is, inhibiting production of A2780 ovarian cancer cells. It is much more efficient in prohibiting the production of A2780 ovarian cancer cells than using only doxorubicin. Therefore, the proposed triangular DNA origami in $120 \mathrm{~nm}$ provides an efficient nanoscale drug delivering systems for inhibiting the reproduction of ovarian cancer cells in vitro with DNA nanotechnology.

For future works, it is of interests to develop novel DNA origami with smaller sizes and acceptable stability to delivery drugs in nanoscale. The balance between the size and stability is the elemental problem. As well, it needs to compare the performance of different DNA origamis in delivering particular drugs.

Bioinformatics focuses on dealing with biological data, such as genome, DNA sequences, RNA and gene networks with computers, see e.g. DNA processing [34], microRNA $[35,36]$. Bio-computing, also known as biomolecular computing, whose purpose is using molecular, DNA or RNA to do computation by constructing molecular level computing devices, such as probe machine [37]. It would be of interests if biological data can be processed by bio-computing devices in the near future.

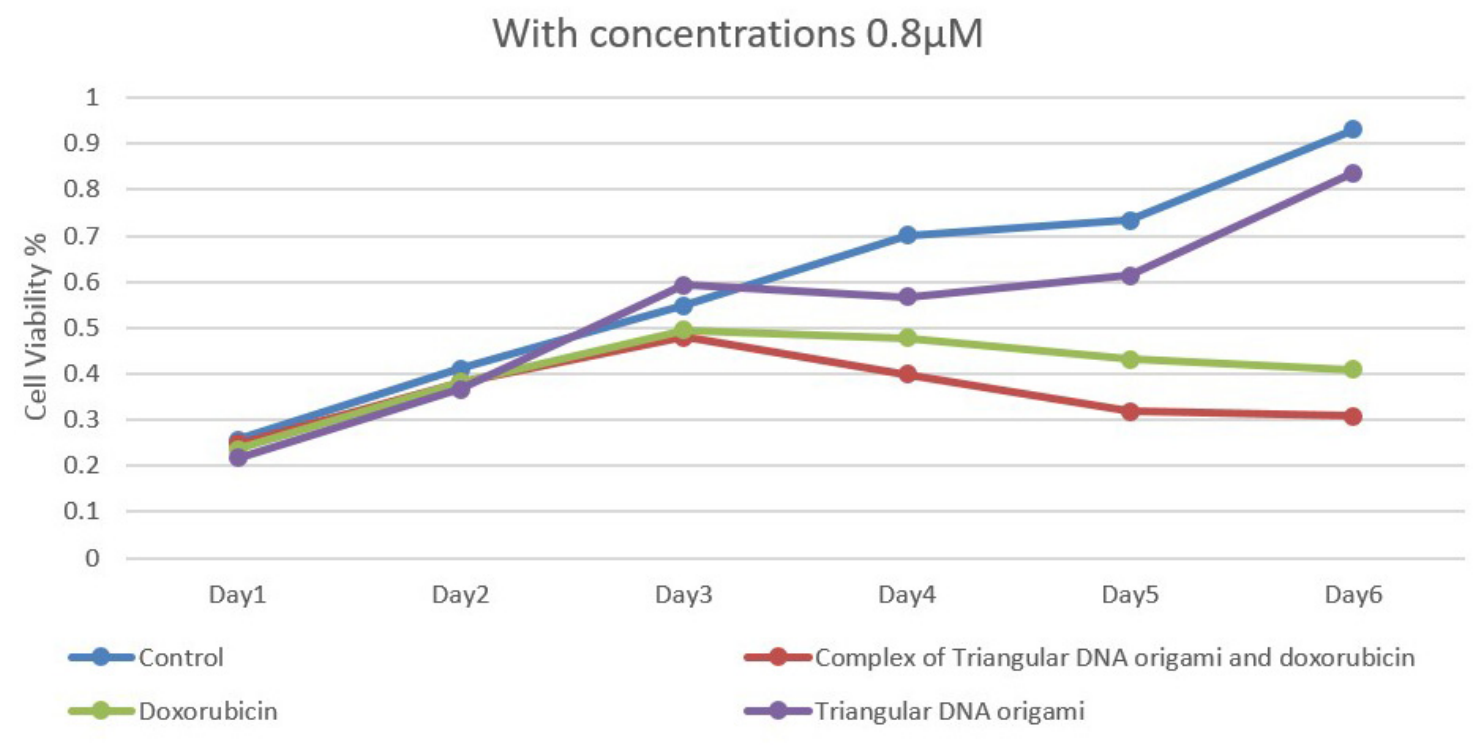

Figure 10: The cell proliferation results by CCK-8 and inhibition of clone formation with concentration $0.8 \mu \mathrm{M}$. 


\section{Author contributions}

Tao Song designed and performed the experiments. Xun Wang developed the ideas of designing triangular DNA origami. Xin Li proposed the idea of targeting A2780 ovarian cancer cells by triangular DNA origami. Pan Zheng gives his help in writing the manuscript. All authors have reviewed and agreed to this information before submission.

\section{CONFLICTS OF INTEREST} interest.

The authors declare that they have no competing

\section{FUNDING}

Tao Song was supported by National Natural Science Foundation of China (61402187 and 61672033), Research Project TIN2016-81079-R(AEI/FEDER, Spain-EU) and grant 2016-T2/TIC-2024 from TalentoComunidad de Madrid. Tao Song was also supported by Research Funds for the Central Universities (16CX02006A). Key Research and Development Program of Shandong Province (No. 2017GGX10147). Xun Wang was supported by National Natural Science Foundation of China (61502535 and 61672248). Xin Li was supported by National Natural Science Foundation of China (61272071, 61672248), Natural Science Foundation of Hubei Province (2014CFB730).

\section{REFERENCES}

1. Seeman NC. DNA in a material world. Nature. 2003; 421: 427-432.

2. Seeman NC. Structural DNA nanotechnology. Cambridge University Press, 2016.

3. Lin C, Yan H. DNA nanotechnology: a cascade of activity. Nat Nanotechnol. 2009; 4: 211-212.

4. Pinheiro AV, Han D, Shih WM. Challenges and opportunities for structural DNA nanotechnology. Nat Nanotechnol. 2011; 6: 763-772.

5. Zhang DY, Seelig G. Dynamic DNA nanotechnology using strand-displacement reactions. Nat Chem. 2011; 3: 103-113.

6. Grakoui A, Bromley SK, Sumen C. The immunological synapse: a molecular machine controlling $\mathrm{T}$ cell activation. Science. 1999; 285: 221-227.

7. Yurke B, Turberfield AJ, Mills AP Jr. A DNA-fuelled molecular machine made of DNA. Nature. 2000; 406: 605.

8. Benenson Y, Gil B, Ben-Dor U. An autonomous molecular computer for logical control of gene expression. Nature. 2004; 429: 423-429.

9. Adleman LM. Molecular computation of solutions to combinatorial problems. Science. 1994; 266: 1021-1024.
10. Shi X, Wu X, Song T, Li X. Construction of DNA nanotubes with controllable diameters and patterns by using hierarchical DNA sub-tiles. Nanoscale. 2016; 8: 14785-14792.

11. Chatterjee G, Dalchau N, Muscat RA. A spatially localized architecture for fast and modular computation at the molecular scale. Nat Nanotechnol. 2017; 12: 920-927. https://doi.org/10.1038/nnano.2017.127.

12. Rothemund P. Folding DNA to create nanoscale shapes and patterns. Nature. 2006; 440: 297-302.

13. Cai L, Friedman N, Xie XS. Stochastic protein expression in individual cells at the single molecule level. Nature. 2006; 440: 358-362.

14. Sanderson K. Bioengineering: what to make with DNA origami. Nature. 2010; 464: 158

15. Yin P, Hariadi RF, Sahu S. Programming DNA tube circumferences. Science. 2008; 321: 824-826.

16. Wei B, Dai M, Yin P. Complex shapes self-assembled from single-stranded DNA tiles. Nature. 2012; 485: 623.

17. Douglas SM, Marblestone AH, Teerapittayanon S. Rapid prototyping of 3D DNA-origami shapes with caDNAno. Nucleic Acids Res. 2009; 37: 5001-5006.

18. Lee KW, Kim KM, Lee J. A two-dimensional DNA lattice implanted polymer solar cell. Nanotechnology. 2011; 22: 375202.

19. Chao J, Huang WY, Wang J. Click-chemistry-conjugated oligo angiomax in the two dimensional DNA lattice and its interaction with thrombin. Biomacromolecules. 2009; 10: 877-883.

20. Dugasani SR, Kim JA, Kim B. A 2D DNA lattice as an ultrasensitive detector for beta radiations. ACS Appl Mater Interfaces. 2014; 6: 2974-2979.

21. Endo M, Hidaka K, Kato T. DNA prism structures constructed by folding of multiple rectangular arms. J Am Chem Soc. 2009; 131: 15570-15571.

22. Hammack A, Chen YL, Pearce JK. Role of dissolved salts in thermophoresis of DNA: lattice-Boltzmann-based simulations. Phys Rev E Stat Nonlin Soft Matter Phys. 2011; 83: 031915.

23. He X, Dong L, Wang W. Folding single-stranded DNA to form the smallest 3D DNA triangular prism. Chem Commun. 2013; 49: 2906-2908.

24. Ke Y, Sharma J, Liu M. Scaffolded DNA origami of a DNA tetrahedron molecular container. Nano Lett. 2009; 9: 2445-2447.

25. An Y, Liu SD, Deng SY. Cleavage of double-strand DNA by linear and triangular trinuclear copper complexes. J Inorg Biochem. 2006; 100: 1586-1593.

26. Ruiz-Hernandez E, Baeza A, Vallet-Regi, M. Smart drug delivery through DNA/magnetic nanoparticle gates. ACS Nano. 2011; 5: 1259-1266. 
27. http://bionano.physics.illinois.edu/tutorials/moleculardynamics-simulation-custom-dna-nanostructures-creatednanoengineer-1.

28. https://cando-dna-origami.org/\#.

29. Mohan P, Rapoport N. Doxorubicin as a molecular nanotheranostic agent: effect of doxorubicin encapsulation in micelles or nanoemulsions on the ultrasound-mediated intracellular delivery and nuclear trafficking. Mol Pharm. 2010; 7: 1959-1973.

30. Cao J, Wang R, Gao N, Li M, Tian X, Yang W, Ruan Y, Zhou C, Wang G, Liu X, Tang S, Yu Y, Liu Y, et al. Gao, A7RC peptide modified paclitaxel liposomes dually target breast cancer. Biomater Sci. 2015; 3: 1545-1554.

31. Davis ME, Chen Z, Shin DM. Nanoparticle therapeutics: an emerging treatment modality for cancer. Nat Rev Drug Discov. 2008; 7: 771.

32. Li M, Yu H, Wang TF, Chang ND, Zhang JQ, Du D, Liu MF, Sun SL, Wang R, Tao HQ, Geng SL, Shen ZY, Wang Q, Peng HS. Tamoxifen embedded in lipid bilayer improves the oncotarget of liposomal daunorubicin in vivo. J Mater Chem B. 2014; 2: 1619-1625.

33. Li X, Hong L, Song T, Rodríguez-Patónc A, Chen C, Zhao H, Shi X. Highly biocompatible drug-delivery systems based on DNA nanotechnology. J Biomed Nanotechnol. 2017; 13: 1-9.

34. Wang $\mathrm{X}$, Miao $\mathrm{Y}$, Cheng M. Finding motifs in DNA sequences using low-dispersion sequences. J Comput Biol. 2014; 21: 320-329.

35. Zeng X, Zhang X, Zou Q. Integrative approaches for predicting microRNA function and prioritizing diseaserelated microRNA using biological interaction networks. Brief Bioinform. 2016; 17: 193-203.

36. Zou Q, Li J, Song L. Similarity computation strategies in the microRNA-disease network: a survey. Brief Funct Genomics. 2015; 15: 55-64.

37. Xu J. Probe machine. IEEE Trans Neural Netw. 2016; 27 : 1405-1411. 